\title{
Ludger Heidbrink
}

\section{Die dunklen Seiten des Konsums: Zusammenfassung und Ausblick}

Der Titel der Jahreskonferenz 2019 - Die dunklen Seiten des Konsums - hatte bei der Vorbereitung ein gewisses Unbehagen hervorgerufen, weil er allzu negativ klang. Im Koordinierungsgremium des Bundesnetzwerks Verbraucherforschung wurde ausgiebig darüber diskutiert, wie die Konferenz inhaltlich ausgerichtet und genannt werden sollte. Uns erschien es angebracht zu sein, über die Schattenseiten des Konsums zu sprechen, um damit auf indirektem Weg zu einer Aufklärung des Verbraucherhandelns beizutragen. Denn wo Schatten ist, muss es auch Licht geben. Die Beleuchtung der dunklen Seiten sollte zu einem besseren Verständnis des Konsums führen. Mit Max Weber gesprochen, könnte man auch sagen, dass auf der Konferenz idealistische Vorstellungen des Konsumverhaltens durch rationale Aufklärung „entzaubert“ (Weber 1995) und auf realistische Grundlagen gestellt werden sollten.

Um die dunkle und die helle Seite des Konsums besser in den Blick zu bekommen, war die Veranstaltung in drei Panels aufgeteilt worden. Im ersten Panel „Zwischen Glücksspiel, Smartphone, Like-Kultur: alte und neue Süchte?" ging es vor allem um Spiel- und Mediensucht. Die Vorträge von Tilman Becker und Tobias Matzner machten deutlich, dass es höchst relevant ist, den Suchtbegriff stärker zu differenzieren. Offensichtlich ist nicht alles, was gemeinhin als Sucht stigmatisiert wird, gleich eine Sucht im klinischen oder pathologischen Sinn. Oft werden gewisse Abhängigkeiten von den KonsumentInnen bewusst eingegangen und sind konform mit dem individuellen Lebensstil und den persönlichen Glücksvorstellungen. Dies muss berücksichtigt werden, um nicht eine frühzeitige oder einseitige Medikalisierung der KonsumentInnen vorzunehmen. Suchtformen im Konsumbereich sind überaus vielfältig und zudem, wie in der Gesprächsrunde deutlich wurde, nicht von vornherein pathologisch. Es sollte also vermieden werden, durch voreilige Suchtdiagnosen die Eigenverantwortung der KonsumentInnen zu vermindern, indem ihnen unterstellt wird, aufgrund ihres Suchtverhaltens nicht frei und selbstbestimmt handeln zu können. Stattdessen ist es wichtig, die Resilienz der KonsumentInnen zu stärken. Hierunter ist die psychische Widerstandskraft gegen schädliche Einflüsse oder Belastungen zu verstehen (vgl. Richter 2017). Maßgeblich ist dabei, dass die Stär- 
kung individueller Widerstandskraft ohne gleichzeitige Beschneidung der Autonomie, etwa durch paternalistische Maßnahmen des Verbraucherschutzes, erfolgt. Die persönliche Freiheit der VerbraucherInnen besteht auch darin, selbstgewählte Abhängigkeiten einzugehen, solange diese die eigene Entscheidungsfähigkeit nicht unterminieren. Die Eigenverantwortlichkeit von KonsumentInnen, so lautet ein erstes Fazit, sollte nicht unterlaufen, sondern gefördert werden, und zwar gerade dort, wo Abhängigkeiten und Süchte entstehen. Falls die Förderung der Eigenverantwortung nicht ausreicht, muss die Resilienzfähigkeit der KonsumentInnen durch rechtliche Regulierungen und eine Verbraucherpolitik unterstützt werden, die immer dann eingreift, wenn VerbraucherInnen ihrer Eigenverantwortung nicht mehr ohne Weiteres nachkommen können. Ein guter Anfang wäre zum Beispiel gemacht, wenn der Gesetzgeber Glücksspieleinrichtungen schließt, die nachweislich die Abhängigkeit vom Glücksspiel erhöhen.

Im zweiten Teil der Konferenz über „VerbraucherInnen und neue Sehnsuchtswelten" standen die medialen Angebote des Bösen und die Auswirkungen der Selbstoptimierung auf das Verbraucherverhalten im Vordergrund. Kaspar Maase zeigte, dass das Böse in Gestalt von neueren Filmen und TV-Serien eine große Faszination auf KonsumentInnen ausübt und einen elementaren Bestandteil der populären Verbraucherkultur bildet. Für die verbraucherpolitische Forschung ergibt sich damit die Herausforderung, in Zukunft auch das deviante und abgründige Verbraucherverhalten genauer in den Blick zu nehmen, das in viel stärkerem Maß, als gemeinhin angenommen wird, eine konsumistische Normalität in der Alltagswelt von VerbraucherInnen darstellt. Die Faszination durch das Böse liegt vor allem darin begründet, dass es imaginäre Gegenwelten zur Verfügung stellt und ein eskapistisches Verhalten befördert, durch das sich MedienkonsumentInnen von der Konfrontation mit realen Nöten und Konflikten entlasten können. Es geht deshalb nicht darum, den Konsum gewalttätiger und bösartiger Filme und Serien moralisch zu verurteilen, sondern das Gute im Gegenzug attraktiver zu machen. Auch das Gute kann faszinierend, spektakulär und überraschend sein, so dass VerbraucherInnen wie beim Bösen durch einen ähnlichen Sog der Wirklichkeitsüberwindung und Realtranszendenz erfasst werden.

Eine ähnliche Faszination wie das Böse üben die Potentiale der Selbstoptimierung aus. Janina Loh setzte sich kritisch mit trans- und posthumanistischen Maßnahmen auseinander, die durch Human Enhancement eine Steigerung der Lebensqualität verfolgen. Der Trans- und Posthumanismus sind philosophische Ideologien, die in der Konsumforschung der Zukunft aller Wahrscheinlichkeit nach eine größere Rolle spielen werden, wenn es etwa um die kommerzielle Optimierung des Menschen durch lebensverlän- 
gernde Technologien, die Potenzierung kognitiver Fähigkeiten oder hybride Mensch-Maschine-Produkte geht. Ebenso stellen körperliche Modifikationen in Kosmetik und Sport sowie Verfahren des Self-Tracking durch digitale Devices Entwicklungen auf den Konsummärkten dar, mit denen sich die Verbraucherforschung in Zukunft befassen muss (vgl. Baule et al. 2019). Wie bei den neuen Konsumsüchten sollte der Wunsch der VerbraucherInnen nach Selbstoptimierung nicht moralisch stigmatisiert werden, sondern als quasi anthropologische Herausforderung betrachtet werden, da Perfektionierungssehnsüchte zur Natur des Menschen zu gehören scheinen.

Im dritten Teil über ,VerbraucherInnen zwischen alten und neuen Egoismen" wurde das verbreitete Phänomen des unmoralischen Konsumentenverhaltens behandelt. Andrea Gröppel-Klein warf in ihrem Vortrag die Frage auf, warum KonsumentInnen zwar zunehmend ethische Ansprüche an den Handel und Unternehmen stellen, beispielsweise in Form von CSR-Standards, selbst aber häufig hinter diesen Ansprüchen zurückbleiben. Rücksichtsloses Konsumverhalten lässt sich etwa dort beobachten, wo VerbraucherInnnen unrechtmäßige Produktrückgabe betreiben, indem sie im Internet gekaufte Kleidung nach Gebrauch als ungenutzt zurücksenden, bei Online-Bestellungen Betrugsmöglichkeiten ausnutzen oder Preisnachlässe durch Falschangaben ergattern. Der gezielte Betrug und die absichtliche Schädigung stellen nicht nur ein volkswirtschaftliches, sondern ein ethisches Problem dar. Sie zeigen, dass KonsumentenInnen verantwortungslos handeln, wenn ihnen Gelegenheiten dazu geboten werden und sie relativ einfach eigene Vorteile erreichen können.

Die Verantwortungslosigkeit von KonsumentInnen, die durch den digitalen Handel forciert wird, eröffnet neue Forschungsfelder: Während das Feld der Corporate Social Irresponsibility (Lange \& Washburn 2012) recht gut untersucht ist, könnte es sinnvoll sein, in Zukunft das Phänomen der Consumer Social Irresponsibility genauer zu erkunden. Die „consumer responsibilization" (Eckhardt \& Dobscha 2018), die in den letzten Jahren im Fokus der verbraucherpolitischen Forschung stand und in Leitbildern wie dem „verantwortlichen Konsumenten“ (vgl. Kenning \& Wobka 2013) zum Ausdruck kommt, wird durch ein unmoralisches Konsumverhalten konterkariert, das im Widerspruch zum bekundeten Selbstverständnis zahlreicher VerbraucherInnen und ihren Erwartungen an die Verantwortung anderer Markteilnehmer steht. Ein möglicher Weg, unmoralischen Verhaltensweisen von VerbraucherInnen entgegenzuwirken, besteht im Abbau von sozialer Distanz bei gleichzeitigem Aufbau von Nähe zwischen den Konsumierenden. Schon Adam Smith hat vom impartial spectator gesprochen, von dem unparteiischen Zuschauer, der bewirkt, dass wir uns mit den „Augen anderer Leute“ (Smith 2004, S. 167) betrachten und auf diese Weise die morali- 
sche Zulässigkeit des eigenen Handelns überprüfen. Ein aktuelles Pendant für den „Spiegel der Anderen“, den Smith im Auge hatte, sind soziale Beobachtungssituationen beim Einkaufen oder digitale Bewertungsportale, die das Bereitschaftspotential von VerbraucherInnen stärken, moralkonform zu agieren, weil sie sich nicht der Missachtung durch andere Marktteilnehmer aussetzen wollen.

Stefan Hoffmann illustrierte in seinem Vortrag, wie vielfältig das Spektrum der KonsumentInnen zur Ablehnung und Leugnung von Verantwortung ist - ein Phänomen, das in der Forschung unter dem Begriff der „denial of responsibility" (Montada 2001) behandelt wird. Anhand empirischer Studien lässt sich zeigen, dass VerbraucherInnnen Virtuosen der Abwehr moralischer Ansprüche sind - entweder durch die Rationalisierung schädlicher Konsumpraktiken, die Entkoppelung moralischer Eigenschaften von ihren Trägern oder die moralische Lizenzierung von Verhaltensweisen: So rücken sich VerbraucherInnen ihren ökologischen Fußabdruck mit fadenscheinigen Argumenten zurecht, drücken bei sozialen Verstößen ihrer Lieblingsmarken häufig ein Auge zu oder gönnen sich eine Kreuzfahrt, nachdem sie täglich mit dem Fahrrad zur Arbeit gefahren sind. Hypokritisches Konsumverhalten stellt zweifellos ein Problem dar, da sich auf diese Weise nichtmoralisches Verhalten entschuldigen lässt, ohne tatsächlich gerechtfertigt zu sein. Allerdings besitzt der scheinheilige Konsument immerhin den Anspruch, das moralisch Richtige zu tun, auch wenn es nicht konsequent umgesetzt wird. Verbraucherpolitisch besteht hier die Möglichkeit, durch Aufklärung und Information die KonsumentInnen dazu zu bringen, sich selbstkritischer, offener und ehrlicher mit ihren Konsumgewohnheiten auseinanderzusetzen.

Zwei weitere Beiträge wurden nach der Jahreskonferenz in diesen Band mit aufgenommen. Martin Klug und Jonas Grauel plädieren vor dem Hintergrund dysfunktionalen Konsumverhaltens für ein neues Leitbild in der Verbraucherpolitik: den „engagierten Verbraucher“. Während dem „unwissenden Verbraucher" grundlegende Einsichten über den Zusammenhang zwischen seinem Handeln und Problemen der Nachhaltigkeit fehlen, der „ignorante Verbraucher" diese Einsichten ausklammert und verdrängt, der „verantwortungsvolle Konsument" regelmäßig hinter seinen Nachhaltigkeitsidealen zurückbleibt, orientiert sich der engagierte Konsument an realistischen Nachhaltigkeitszielen und ist bemüht, diese auch tatsächlich umzusetzen. Der engagierte Konsument, der Repair Cafés aufsucht, Urban Gardening betreibt und Zero Waste-Lebensstile praktiziert, stellt eine pragmatische Vorstufe auf dem Weg zum verantwortungsvollen Verbraucher dar. Er bildet nach Meinung der Autoren das empirisch fundierte Leitbild für eine 
differenzierte Verbraucherpolitik, die den nachhaltigen Konsum effektiv befördern will.

Der Beitrag von Julian Nida-Rümelin und Nathalie Weidenfeld über „Ökonomische Rationalität als Softwareprogramm“ macht abschließend deutlich, dass digitale Technologien, die auch im Alltagskonsum immer mehr Anwendung finden, sich zu eigendynamischen Systemen entwickeln können, die von menschlicher Verantwortung abgekoppelt sind. Die Folge wäre eine inhumane Wirtschaft, in der nicht menschliche Bedürfnisse im Vordergrund stehen, sondern ökonomische Nutzenkalküle. Um dieses Risiko zu vermeiden, müssen digitale Optimierungsprogramme menschlicher Zwecksetzung unterworfen bleiben sowie durch Werte der Gerechtigkeit und Solidarität bestimmt werden.

Welcher Ausblick auf die Konsumforschung und Verbraucherpolitik ergibt sich aus der Konferenz und den Beiträgen dieses Bandes? Sie zeigen vor allem, dass dysfunktionale und unmoralische Verhaltensweisen zur Normalität des Alltagskonsums gehören. KonsumentInnen unterliegen in ihren Entscheidungen situativen Einflüssen, haben sich nicht immer unter Kontrolle, agieren impulsiv und irrational, verfolgen ihre eigenen Interessen und reden sich ihr Fehlverhalten schön. Hieraus ergeben sich drei Herausforderungen für die zukünftige Konsumforschung und Verbraucherpolitik:

Zum Einen ist umfassender als bisher eine ambivalente Beurteilung des Konsumverhaltens erforderlich. Die meisten Konsumentscheidungen sind nicht eindeutig, sondern mehrdeutig. Im Alltag verfolgen VerbraucherInnen unterschiedliche Ziele zugleich. Mehrdeutigkeit des Konsums bedeutet, dass altruistische und egoistische Motive parallel auftreten können, das gute und das schlechte Handeln nebeneinander stattfinden. Die dunkle Seite ist kein Spezialfall oder Sonderphänomen des Alltagskonsums, sondern tritt regelmäßig in Erscheinung, wenn Güter und Dienstleistungen konsumiert oder persönliche Bedürfnisse umgesetzt werden, so dass eine vorschnelle Kritik dysfunktionalen und unmoralischen Verhaltens in vielen Fällen Fehl am Platz ist.

Dies führt zweitens zu einer moralischen Relativierung des Konsumverhaltens. KonsumentInnen handeln nicht besser als andere Marktteilnehmer, sie sehen sich aber häufig so und klagen gerne die Unmoral der anderen an. In Umfragen und Selbstbekundungen überwiegen eine positive Eigenwahrnehmung und der Wille zu nachhaltigem und sozialem Handeln. Im alltäglichen Leben und bei der Zahlungsbereitschaft bleiben die KonsumentInnen aber häufig hinter ihren artikulierten und intendierten Zielen zurück. Die Kluft zwischen Einstellungen und Verhalten, der mind behaviour gap, ist ein altes Problem, das sich besser verstehen lässt, wenn man beispielsweise vom Typus des hypokritischen Konsumenten ausgeht, der zwar die Bereit- 
schaft zum richtigen Handeln besitzt, aber nicht über hinreichend starke Motive verfügt, sein Selbstbild auch tatsächlich zu realisieren. Die Einsicht in die eigene Schwäche und mangelnde Handlungskonsequenz kann dabei helfen, die verfolgten Ziele konsequenter zu erreichen. Indem VebraucherInnen ihre Selbstwahrnehmung moralisch relativieren und faktische Defizite in ihrem Verhalten erkennen, sind sie eher in der Lage, sich realistische Konsumziele zu setzen, deren Verfehlung nicht wieder zu Strategien der moralischen Rationalisierung und der Leugnung von Verantwortlichkeit führt.

Damit ergibt sich zum Dritten die Herausforderung, ein verbraucherpolitisches Leitbild zu finden, mit dem sich das Phänomen der Consumer Social Irresponsibility adäquat erfassen lässt, ohne dabei weder die helle noch die dunkle Seite des Konsumverhaltens überzubetonen. Als Ergänzung zum verantwortlichem, vertrauenden und verletzlichen Verbraucher (Micklitz et al. 2010) könnte sich dafür der unverantwortliche Verbraucher eignen. Der unverantwortliche Verbraucher ist etwas anderes als der unwissende oder ignorante Verbraucher, der nicht über das erforderliche Wissen verfügt oder dieses absichtlich nicht berücksichtigt, wie Martin Klug und Jonas Grauel in ihrem Beitrag gezeigt haben. Im Unterschied zum unwissenden und ignoranten Verbraucher weiß der unverantwortliche Konsument, dass sein Handeln moralischen, sozialen oder rechtlichen Kriterien nicht entspricht und sucht deshalb nach Gründen, mit denen er sein Fehlverhalten erklären kann. Der unverantwortliche Konsument bewegt sich in einer AmbivalenzZone zwischen einer legitimen Unverantwortlichkeit, etwa dann, wenn notwendige Informationen fehlen oder moralische Kosten zu hoch sind, und einer illegitimen Verantwortungslosigkeit, beispielsweise dort, wo betrügerisch agiert wird oder zumutbare Nachhaltigkeitsalternativen ausgeschlagen werden (vgl. Heidbrink 2015).

Das Leitbild des unverantwortlichen Verbrauchers entspricht der Mehrdeutigkeit des Alltagskonsums, der in der Regel auf einer Überlagerung divergenter Motive beruht, die wohlbegründet, irrtümlich entstanden oder bloß vorgetäuscht sein können. Das Leitbild des unverantwortlichen Verbrauchers lässt offen, ob KonsumentInnen tatsächlich keine Verantwortung tragen oder sich absichtlich der Verantwortung entziehen wollen, die sie bei genauerer Betrachtung besitzen. Für die Verbraucherpolitik kann ein solches Leitbild hilfreich sein, da es nicht vorgibt, welche Art und welcher Umfang der Verantwortung den KonsumentInnen zugeschrieben werden soll. Es macht vielmehr die Zuschreibung der Verantwortung von konkreten Handlungssituationen und marktlichen Entscheidungskontexten abhängig, die fallspezifisch beurteilt werden müssen. Insofern bringt die dunkle Seite 


\section{des Konsums am Ende auch Licht in die Schattenwelt der Konsumentenver- antwortung.}

\section{Literatur}

Baule, B., Hohnsträter, D., Krankenhagen, S. \& Lamla, J. (Hrsg.) (2019). Transformationen des Konsums. Vom industriellen Massenkonsum zum individualisierten Digitalkonsum. Baden-Baden: Nomos.

Eckhardt, G.M. \& Dobscha, S. (2018). „The Consumer Experience of Responzibilization: The Case of Panera Cares“. In: Journal of Business Ethics, https://doi.org/10.1007/s10551-018-379 5-4.

Heidbrink, L. (2015). „Verantwortung und Verantwortungslosigkeit“. In: K.P Liessmann (Hrsg.). Schuld und Sühne. Nach dem Ende der Verantwortung. Wien: Zolnay, S. 186-207.

Lange, D. \& Nathan, W. (2012). „Understanding Attributions of Corporate Social Irresponsibility“. In: Academy of Management Review, Vol. 37, No. 2, S. 300-326.

Micklitz, Hans-W., Oehler A., Piorkowsky, M.-B., Reisch, L. C., \& Strünck, C. (2010). Der vertrauende, der verletzliche oder der verantwortungsvolle Verbraucher? Stellungnabme des Wissenschaftlichen Beirats für Verbraucher- und Ernährungspolitik beim BMELV. Berlin.

Montada, L. (2001). „Denial of Responsibility“. In: A. E. Auhagen \& H.-W. Bierhoff (Hrsg.). Responsibility. The Many Faces of a Social Phenomenon, London/New York: Routledge, S. 79-92.

Kenning, P. \& Wobker, I. (2013). „Ist der 'mündige Verbraucher' eine Fiktion? Ein kritischer Beitrag zum aktuellen Stand der Diskussion um das Verbraucherleitbild in den Wirtschaftswissenschaften und der Wirtschaftspolitik". In: Zeitschrift für Wirtschafts- und Unternehmensethik, Jg. 14, Heft 2, S. 282-300.

Richter, C. (Hrsg.) (2017). Ohnmacht und Angst aushalten. Kritik der Resilienz in Theologie und Philosophie. Stuttgart: Kohlhammer.

Smith, A. (2004). Theorie der ethischen Gefühle. Hamburg: Meiner.

Weber, M. (1995). Wissenschaft als Beruf. Stuttgart: Reclam. 
